\title{
Evaluation of lightning induced over-voltage due to variations of channel angle
}

\begin{abstract}
Lightning can create lightning induced overvoltage (LIOV) on a power line. However, detailed considerations are needed concerning the evaluation behaviour of LIOV due to changes in channel angle which is referred to as an inclined lightning channel. The aim of this paper is to evaluate the behaviour of LIOV due to variations of channel angle. The LIOV was generated through the execution of an algorithm in MATLAB. Results indicate that the behaviours of LIOV are direct and show a linear relationship with an increasing channel angle which is an inclined angle and observation point angle. On the other hand, the effect on the LIOV of an increasing channel angle due to the return stroke velocity decreases at the highest velocity. As a result, the outcome of this paper could be useful for electrical power designers for the fast estimation of LIOV peak voltages without recalculation of the LIOV and to assist in the appropriate planning of protection schemes.
\end{abstract}

Keyword: Basic Insulation Level; Critical flash over; Inclined angle; Inclined lightning channel; Lightning induced overvoltage; Observation point angle; Velocity of return stroke 